\title{
Improved Quality and Diagnostic Confidence Achieved by Use of Dose-Reduced Gadolinium Blood-Pool Agents for Time-Resolved Intracranial MR Angiography
}

S. Dehkharghani, J. Kang, and A.M. Saindane

\begin{abstract}
BACKGROUND AND PURPOSE: Time-resolved MRA with the use of bolus injection of paramagnetic agents has proved valuable in neurovascular imaging. Standard contrast agents have limited blood-pool residence times, motivating the development of highly protein-bound blood-pool agents with greater relaxivity and longer intravascular residence, affording improved image quality at lesser doses. This study represents the first comparison of blood-pool agents to standard agents in time-resolved cerebral MRA.
\end{abstract}

MATERIALS AND METHODS: One hundred datasets were acquired at $1.5 T$ by use of a standardized, time-resolved MRA protocol. Patients received either unit dosing of a standard extracellular agent at $0.1 \mathrm{mmol} / \mathrm{kg}$ or a blood-pool agent at $0.03 \mathrm{mmol} / \mathrm{kg}$. Peak arterial and venous enhancement phases were identified and subsequently scored qualitatively by use of a 4-point Likert scale, with attention to 6 vascular segments: 1) intracranial ICA; 2) MCA M1; 3) MCA M2; 4) MCA M3; 5) deep cerebral veins; and 6) dural venous sinuses.

RESULTS: Fifty MR angiographies were acquired with each agent. No significant differences were found between agents in generation of uncontaminated arteriograms. Blood-pool agents, at $67 \%$ dose reduction, were of significantly greater quality across most vascular segments, including ICA ( $P=.019)$, M2 $(P=.003)$, and M3 $(P<.01)$. Superiority in the M1 segment approached significance $(P=.059)$. Significantly better venographic quality was noted for deep venous structures $(P=.016)$ with the use of blood-pool agents.

CONCLUSIONS: Blood-pool agents provide superior demonstration of most intracranial vessels in time-resolved MRA compared with standard agents, at reduced doses. The greater relaxation enhancement and more favorable dosing profile make blood-pool agents superior to standard agents for use in cerebral time-resolved MRA.

ABBREVIATIONS: TR-MRA = time-resolved MRA; BPA = blood-pool agents; $\mathrm{SCA}=$ standard contrast agents; DAVF = dural arteriovenous malformation

$\mathrm{T}_{\mathrm{p}}^{\mathrm{i}}$ ime-resolved MRA (TR-MRA) techniques have shown great potential as noninvasive approaches to probing vascular flow in a temporally sensitive manner. ${ }^{1-3}$ Efforts to rapidly image contrast kinetics have benefited from acquisition schemes aimed at accelerated data collection, primarily through the use of parallel receive algorithms and novel $k$-space trajectories. ${ }^{1,3,4}$ Inherent to most time-resolved techniques is the intravenous bolus injection of paramagnetic agents, providing relaxation enhancement of

Received April 5, 2013; accepted after revision June 7.

From Neuroradiology Division, Department of Radiology and Imaging Sciences (S.D., A.M.S.), Emory University Hospital, Atlanta, Georgia; and Department of Biostatistics and Bioinformatics (J.K.), Rollins School of Public Health, Emory University, Atlanta, Georgia.

Abstract previously presented at: Annual Meeting of the American Society of Neuroradiology, May 20-23, 2013; San Diego, California.

Please address correspondence to Seena Dehkharghani, MD, Neuroradiology Division, Department of Radiology and Imaging Sciences, Emory University Hospital, 1364 Clifton Rd, NE, Atlanta, GA 30322; e-mail: seena.dehkharghani@gmail.com

- Indicates open access to non-subscribers at www.ajnr.org

http://dx.doi.org/10.3174/ajnr.A3693 blood T1. ${ }^{1,5}$ Growing concerns regarding the safety profile of such agents, particularly in the setting of renal insufficiency, can limit their widespread use. ${ }^{6}$ The small molecular size of many gadolinium-based vascular contrast agents results in limited bloodpool residence times; thus, the motivation for development of blood-pool agents (BPA) exhibiting higher protein binding and prolonged vascular dwelling. ${ }^{4,7-9}$ Some BPA formulations have been observed to augment $\mathrm{T} 1$ relaxation enhancement at $1.5 \mathrm{~T}$ in water, plasma, and, blood, owing to the multiple paramagnetic ions attached to each macromolecule. ${ }^{9}$ Their pharmacokinetic profiles may therefore afford flexibility in allowing for smaller administered doses at theoretically comparable diagnostic quality.

The first BPA, gadofosveset trisodium (Vasovist; Bayer Schering Pharma, Berlin, Germany_European, Asian rights; Lantheus Medical Imaging, Billerica, Massachusetts-North American, Australian rights) was approved for use in the United States in 2008, and previously in Europe in 2005. ${ }^{4}$ By comparison to the 100 -second distribution phase half-life of standard extracellular 
Table 1: Analysis of enhancement kinetics: BPA versus SCA

\begin{tabular}{lccc}
\multicolumn{1}{c}{ Category } & BPA & SCA & P Value \\
\hline${\text { Presence of uncontaminated arterial phase, }{ }^{\mathrm{a}} \%}^{\text {\% }}$ & $68 \pm 47$ & $68 \pm 47$ & 1 \\
Peak arterial phase $^{\mathrm{b}}$ (sub) & $6.96 \pm 1.63$ & $6.15 \pm 1.84$ & .023 \\
Peak arterial phase $^{\mathrm{b}}$ (unsub) & $6.96 \pm 1.63$ & $6.15 \pm 1.84$ & .023 \\
Peak venous phase $^{\mathrm{b}}$ (sub) & $8.52 \pm 1.64$ & $8.0 \pm 1.68$ & .123 \\
Peak venous phase $^{\mathrm{b}}$ (unsub) & $8.52 \pm 1.64$ & $8.0 \pm 1.68$ & .123 \\
Peak venous-peak arterial phase $^{\mathrm{b}}$ (sub) & $1.56 \pm 1.07$ & $1.88 \pm 1.19$ & .161 \\
Peak venous-peak arterial phase $^{\mathrm{b}}$ (unsub) & $1.56 \pm 1.07$ & $1.86 \pm 1.18$ & .186 \\
\hline
\end{tabular}

Note:-All values are reported as mean \pm standard deviation; sub, unsub reflect background subtracted and nonsubtracted volumes.

a Percentage of cases with uncontaminated arteriographic phase (see text).

${ }^{b}$ Expressed in terms of average, time-resolved phase number.

gadolinium chelates, gadofosveset achieves a mean initial plasma half-life of 29 minutes in the distribution phase through reversible albumin binding. ${ }^{10-12}$

Past studies have investigated the use of BPA for steady-state MRA, with attention to the theoretic advantages of prolonged vascular residence for high-resolution angiography; however, more recent investigations have demonstrated the advantages of BPA for TR-MRA, exploiting their profile for peripheral and thoracic first-pass MRA and focused imaging during the equilibrium phase. ${ }^{4,13-17}$ We propose to compare the qualitative efficacy of BPA with a low protein-bound, standard contrast agent (SCA) in time-resolved cerebral MR angiography.

\section{MATERIALS AND METHODS Imaging Protocol}

One hundred TR-MRA datasets divided equally between BPA and SCA were acquired on clinical $1.5 \mathrm{~T}$ systems (Signa HDxH; GE Healthcare, Milwaukee, Wisconsin) by use of body transmit and signal reception with a dedicated 8-channel head coil. Patients were selected continuously from an electronic query of the radiology informatics system for the primary field of time-resolved brain MRA between November 2010 and September 2012. Institutional review board approval was obtained for this investigation. The administered agent was selected arbitrarily at the time of examination and without a predefined or set pattern of prescription. Most patients underwent TR-MRA for either known or suspected aneurysm, known or suspected dural arteriovenous malformation (DAVF), aneurysm after treatment (after either endovascular coil or Pipeline Embolization Device [Covidien, Irvine, California]), or DAVF after embolization. Imaging evaluation included a product iteration of $3 \mathrm{D}$ time-resolved imaging of contrast kinetics $\left(\mathrm{TR}=4.5 \mathrm{~ms}\right.$; $\mathrm{TE}=\mathrm{min}$; flip angle $=35^{\circ}$; frequency field of view $=17 \mathrm{~cm}$; matrix $=256 \times 160$ zero-filled and interpolated to $512 \times 512$; averages, 0.75 ; bandwidth, $62.5 \mathrm{KHz}$; array spatial sensitivity encoding technique acceleration factor $=$ 2) yielding 10 or 12 dynamic, temporally discrete postcontrast phases at approximately 4.0-second temporal update.

TR-MRA in all patients was performed after the injection of either a low protein-bound SCA gadobenate dimeglumine (MultiHance; Bracco Diagnostics, Princeton, New Jersey), prescribed at $0.1 \mathrm{mmol} / \mathrm{kg}$ and $2 \mathrm{~mL} / \mathrm{s}$ injection rate, or the $\mathrm{BPA}$ gadofosveset trisodium at $0.03 \mathrm{mmol} / \mathrm{kg}$ and $1.5 \mathrm{~mL} / \mathrm{s}$ injection rate. Slower injection rates for BPA were used to ameliorate seldom-reported midsection burning and discomfort at higher rates as well as to optimize the biophysical profile of gadofosveset relaxation enhancement at $1.5 \mathrm{~T}$ (see Discussion section). Bolus infusion in both protocols was followed immediately by $25-30$ $\mathrm{mL}$ normal saline flush at $2 \mathrm{~mL} / \mathrm{s}$. Postinjection delay and sequence triggering were prescribed in an automated fashion, without user input, by the scanner/sequence manufacturer. The dynamic data generated by the time-resolved imaging of contrast kinetics sequence are presented in numbered temporal datasets. The scan baseline reflects the simultaneous initiation of the sequence and initiation of contrast injection, both occurring under fully automated parameters prescribed by the scanner/sequence manufacturer, and without user input. Before contrast/ sequence triggering, a noncontrast mask is acquired for subtraction. Background subtraction of the precontrast imaging volume was performed in-line during acquisition, allowing for production of both background-subtracted and unsubtracted TR-MRA volumes.

\section{Image Analysis}

All data were analyzed by the consensus evaluation of 2 neuroradiologists, both with subspecialty certification in neuroradiology. Both readers were blinded to the administered agent at the time of review. Evaluation was performed as follows: for each examination, background-subtracted and unsubtracted maximum intensity projection volumes (temporal frames) were simultaneously evaluated for identification of the temporal phase displaying peak arterial and venous enhancement. Any temporal discordance between the 2 volumes was recorded, and the presence or absence of an uncontaminated (ie, free of venous enhancement) angiographic phase was specifically noted for each case. Peak enhancement phases were determined from the proximal branches of the circle of Willis and transverse sinuses for arterial and venous phases, respectively, and numeric temporal phase was recorded for analysis. Peak enhancement phases and qualitative analysis of TR-MRA (see below) was generally assessed by inspection of the bilateral anatomy; however, for cases in which large vascular abnormalities, shunt physiology, or artifacts related to stent placement confounded this methodology, characterization was limited to the disease-free or untreated side.

After determination of peak arterial and venous phases, the corresponding axial source volumes were selected for further qualitative analysis. For both phases, subtracted and unsubtracted source data were synchronized to allow for the simultaneous analysis of TR-MRA quality, with attention to 6 vascular segments: 1) cavernous and supraclinoid ICAs; 2) MCA M1 segment; 3) MCA M2; 4) MCA M3; 5) deep venous system (vein of Galen, internal cerebral veins, thalamostriate veins); and 6) superficial venous system (transverse, sigmoid, and superior sagittal sinuses). The above vascular segments were inspected and scored by the following Likert-type scale proposed by Frydrychowicz et $\mathrm{al}^{13}: 0=$ severely limited, nondiagnostic examination; 1 = fair, diagnosis possible but limited; 2 = good, diagnosis readily possible; and $3=$ excellent diagnostic quality. 

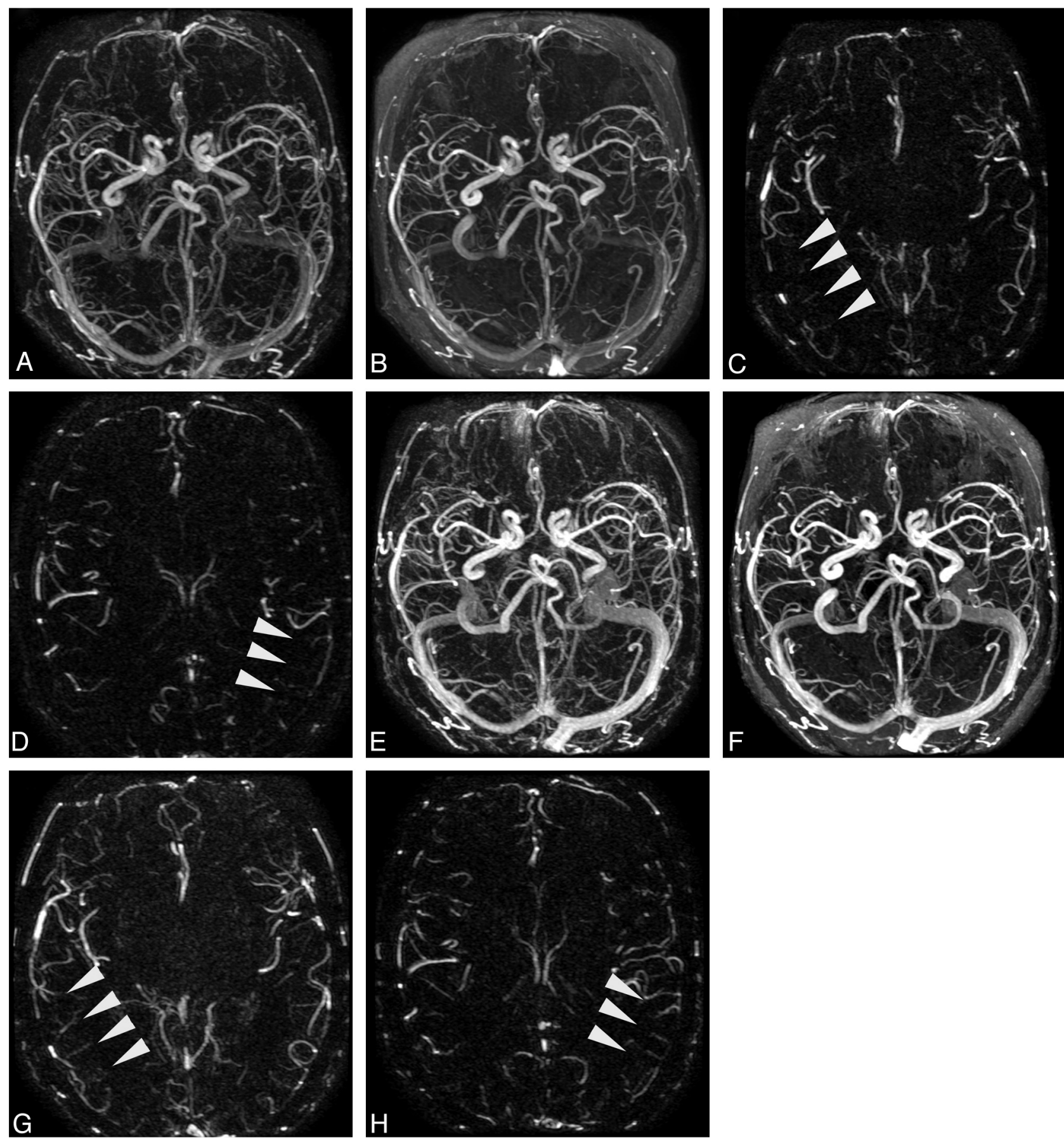

FIG 1. Patient (71-year-old man) undergoing successive TR-MRA, spaced approximately 1 year apart, for evaluation of previously coiled left ophthalmic artery aneurysm. Images obtained after injection of SCA $(A-D)$ and obtained with BPA $(E-H)$. Background-subtracted $(A$ and $E)$ and unsubtracted $(B$ and $F)$ axial MIP volumes from SCA and BPA TR-MRA, respectively; $C, G, D$, and $H$ are background-subtracted axial source MRA images from peak arterial phases derived from SCA (phase 7) and BPA (phase 9) examinations. Note the improved demonstration of mid and distal MCA branches in both hemispheres with administration of the blood-pool agent.

Images were further characterized for artifact-related degradation, including motion, ghosting, and residual aliasing or other artifacts related to parallel acceleration or the view sharing process. Scoring for artifactual degradation was performed as follows: $0=$ severe degradation precluding diagnosis; 1 = moderate artifacts, diagnosis possible but limited; 2 = minor artifacts not affecting diagnosis; and $3=$ no artifacts. Binary assessment of background subtraction quality for each case was rendered as satisfactory or unsatisfactory.

\section{Statistical Analysis}

Categoric data, including the binary determination of the presence versus absence of an uncontaminated arteriographic phase-specifically if the peak arteriographic phase was uncontaminated-as well as the presence or absence of satisfactory background suppression were assessed by Fisher exact test. Phase of peak arterial and peak venous enhancement, as well as the qualitative analysis of vessel segments and artifactual degradation, were treated as continuous variables, and analyzed by Student $t$ test. 
Table 2: Qualitative analysis of image quality: BPA versus SCA

\begin{tabular}{lccc}
\hline & BPA $^{\mathbf{a}}$ & SCA $^{\mathbf{a}}$ & $\boldsymbol{P V a l u e}$ \\
\hline Arterial segment & & & .019 \\
ICA (sub) & $2.74 \pm 0.56$ & $2.42 \pm 0.76$ & .002 \\
ICA (unsub) & $2.88 \pm 0.33$ & $2.56 \pm 0.61$ & .427 \\
M1 (sub) & $2.60 \pm 0.67$ & $2.50 \pm 0.58$ & .059 \\
M1 (unsub) & $2.80 \pm 0.45$ & $2.62 \pm 0.49$ & .015 \\
M2 (sub) & $2.38 \pm 0.75$ & $2.00 \pm 0.78$ & .003 \\
M2 (unsub) & $2.62 \pm 0.57$ & $2.24 \pm 0.69$ & $<.001$ \\
M3 (sub) & $2.18 \pm 0.77$ & $1.56 \pm 0.93$ & $<.001$ \\
M3 (unsub) & $2.44 \pm 0.58$ & $1.76 \pm 0.92$ & .650 \\
Venous segment & & & .562 \\
Superficial (sub) & $2.94 \pm 0.24$ & $2.96 \pm 0.20$ & \\
Superficial (unsub) & $2.98 \pm 0.14$ & $2.96 \pm 0.2$ & .037 \\
Artifactual degradation & & & .016 \\
Deep (sub) & $2.80 \pm 0.4$ & $2.60 \pm 0.53$ & .781 \\
Deep (unsub) & $2.92 \pm 0.27$ & $2.74 \pm 0.44$ & .473 \\
Subtracted & $2.78 \pm 0.55$ & $2.74 \pm 0.53$ & .172 \\
$\quad$ Unsubtracted & $2.82 \pm 0.44$ & $2.74 \pm 0.53$ & \\
Subtraction & & & \\
Subtraction & $0.98 \pm 0.14$ & $0.92 \pm 0.27$ & \\
\hline
\end{tabular}

Note:-All values are reported as mean \pm standard deviation; 0 indicates nondiagnostic; 1, diagnosis possible but limited; 2, good; 3, excellent; sub, unsub, subtracted and nonsubtracted.

${ }^{a}$ Qualitative designation of diagnostic quality derived from 4-point scale (see text).

${ }^{b}$ Indicates percentage of cases for which background subtraction was satisfactory.

\section{RESULTS}

Ninety-two patients (69 women, 23 men; age, 16-97 years; median $=61.5$ years $)$ constituted the study population. Eight patients underwent multiple scans; specifically, 1 patient underwent 4 TR-MR angiographies ( 3 with SCA, 1 with BPA), 5 patients underwent 2 TR-MRA ( 1 each with SCA and BPA), and 2 patients underwent scanning twice with SCA. No subjects received BPA on more than a single scanning session. All available scans for all patients within the accrual period were analyzed, irrespective of administered agent.

All datasets were complete and deemed to be satisfactory for assessment. Patients undergoing scanning with SCA were examined for the following reasons: known or suspected aneurysm $(n=8)$; aneurysm imaging status after coil embolization $(n=$ $22)$; vessel imaging status after Pipeline stent placement $(n=3)$; known or suspected DAVF $(n=7)$; DAVF imaging status after endovascular therapy $(n=5)$; and miscellaneous (including dizziness, dementia, and tumor imaging) $(n=5)$. Among patients undergoing scanning with BPA, indications included known or suspected aneurysm $(n=18)$; aneurysm imaging status after coil embolization $(n=10)$; aneurysm imaging status after surgical clipping $(n=1)$; vessel imaging status after Pipeline stent placement $(n=6)$; known or suspected DAVF $(n=7)$; DAVF imaging status after endovascular therapy $(n=6)$; and miscellaneous (including hypertension and tumor) $(n=2)$.

Significant differences were not found between agents in their likelihood of generating uncontaminated arterial phase volumes (Table 1), observed for both agents in approximately $68 \%$ of examinations. Despite this similarity, significantly delayed peak arterial phase enhancement (Fig 1) was observed for both the subtracted and unsubtracted BPA angiograms (expressed in terms of numeric phase) as compared with SCA (6.96 \pm 1.63 versus $6.15 \pm$ 1.84 , respectively; $P=.023$ ). Minor differences in peak venous enhancement between the agents did not reach statistical significance.
BPA angiograms were of generally greater quality across all interrogated vascular segments (Table 2), with average rating compared with SCA on unsubtracted volumes as follows: ICA $=$ $2.88 \pm 0.33$ versus $2.56 \pm 0.61, P=.002$; $\mathrm{M} 2=2.62 \pm 0.57$ versus $2.24 \pm 0.69$, $P=.003 ; \mathrm{M} 3=2.44 \pm 0.58$ versus $1.76 \pm 0.92, P<.001$ (Fig 2). A trend toward superiority of BPA, not reaching statistical significance, was observed for the M1 segment of the MCA: $2.8 \pm 0.45$ versus $2.62 \pm 0.49, P=.059$; subtracted volumes similarly showed significantly improved quality on BPA angiograms for most segments.

Whereas significant differences in quality were not identified between the agents for characterization of the superficial venous segments, venography of the deep system demonstrated significant superiority of BPA over SCA on subtracted and unsubtracted volumes (unsubtracted $2.92 \pm 0.27$ versus $2.74 \pm 0.44, P=.016$; subtracted $2.8 \pm 0.4$ versus $2.6 \pm 0.53, P=.037$, respectively).

Artifactual degradation and the quality of subtraction did not differ significantly between the 2 populations (Table 2 ).

\section{DISCUSSION}

The present study confirms the feasibility and qualitative superiority of the BPA gadofosveset disodium over the SCA gadobenate dimeglumine for use in time-resolved cerebral MRA. The findings suggest that several theoretic advantages to the use of BPA for such CNS applications may be fully realized when dosing strategies and delivery are optimized. Specifically, the higher proteinbinding capacity of BPA ( $80-96 \%$ reversible albumin binding), together with augmented $\mathrm{T} 1$ relaxation enhancement at clinical field strengths $(\mathrm{r} 1=19$ versus $\mathrm{r} 1=6.3$ at $1.5 \mathrm{~T})$ increases the duration of the available acquisition window and provides improved vascular-to-background contrast, respectively. ${ }^{9}$ Furthermore, the benefits of greater T1 relaxivity afford flexibility in dosing strategies, as demonstrated by the $70 \%$ dose reduction $(0.03$ mmol versus $0.1 \mathrm{mmol}$ ) in our implementation, notably at uniformly improved angiographic quality. Given the superiority of gadobenate over many other extracellular SCA, including its greater relaxivity and its transient protein interaction, we believe that our findings may be readily generalizable to many other SCA as well. ${ }^{9,18,19}$

Although the benefits of BPA for use in high-resolution, steady-state MRA were the subject of earlier studies, more recent reports have highlighted their potential advantages for use in nonCNS TR-MRA. ${ }^{4,13-17}$ A number of vendor-based approaches to accelerated time-resolved imaging have been introduced, generally coupling some combination of parallel acceleration and variable $k$-space sampling attenuation with temporal interpolation and view sharing. ${ }^{1,3,20}$ While facilitating the timing of contrast-enhanced MRA and approximating catheter angiographic 

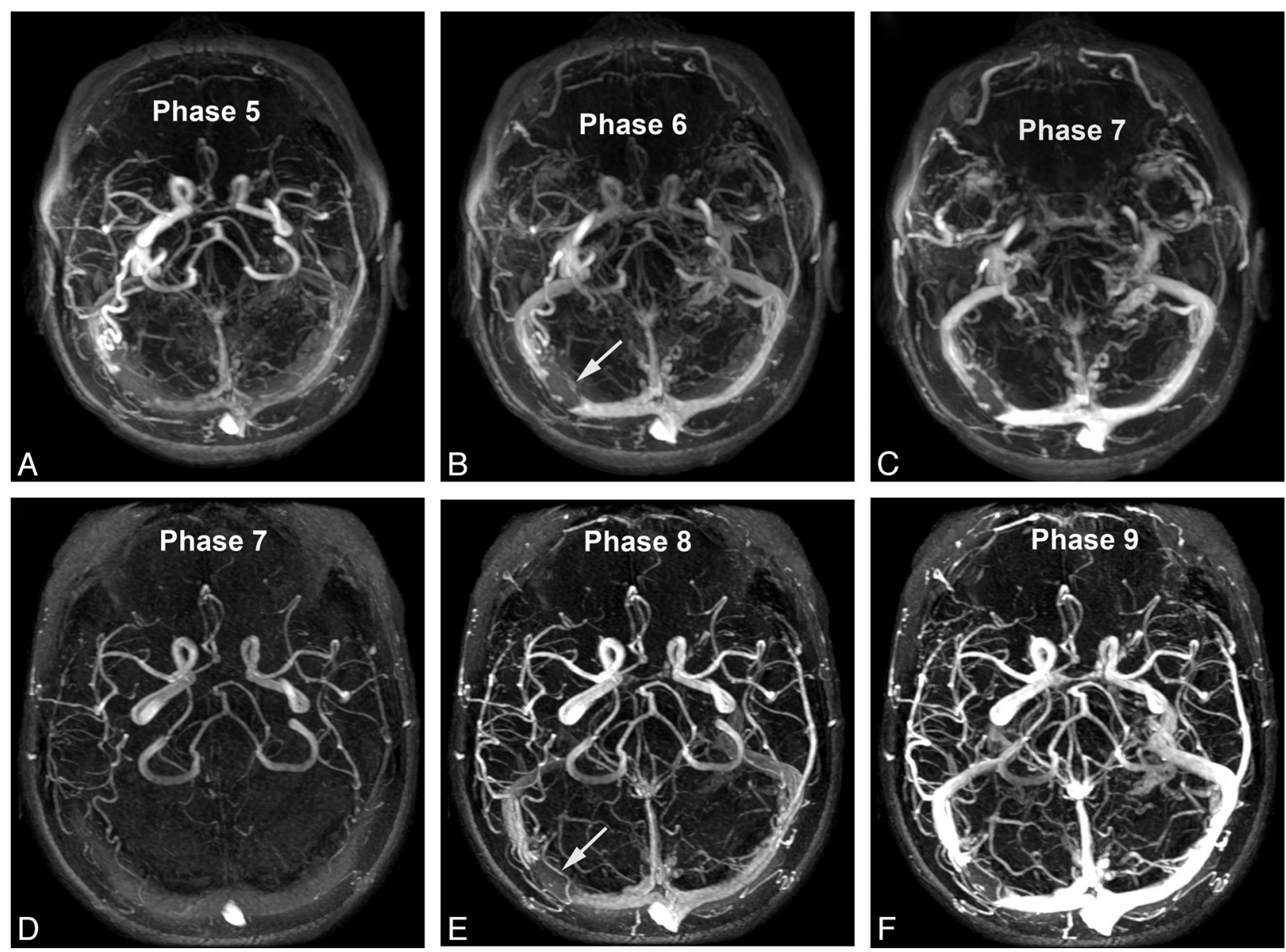

FIG 2. Patient (68-year-old woman) undergoing successive TR-MRA spaced approximately 1 year apart, for evaluation of angiographically documented indirect dural arteriovenous fistula (arrow). Images in $A-C$ were obtained with injection of SCA; images in $D-F$ were obtained with BPA. Early arterial $(A, D)$, late arterial $(B, E)$, and venous $(C, F)$ axial MIP phases are presented as indicated. Qualitatively superior time-resolved angiography was scored for all temporal phases and arterial segments, with consideration to diagnostic confidence and vessel-background contrast. Note the generally delayed arrival/temporal phases with the slower administration rate of BPA.

physiologic information, such approaches may be prone to various degrees of temporal smearing, more common with traditional keyhole undersampling algorithms, which may confound interpretation of dynamic information when severe. We have not observed these effects to be particularly problematic in most cases; however, practitioners should remain aware of their potential to mimic shunt physiology and premature venous enhancement.

We have adopted a slower injection rate for BPA than that used in a similar, recent study by Frydrychowicz et al ${ }^{13}$ comparing BPA with SCA in thoracic TR-MRA $(1.5 \mathrm{~mL} / \mathrm{s}$ versus $3 \mathrm{~mL} / \mathrm{s}$, respectively). In combination with other factors, this difference may be nontrivial, given the nearly uniform superiority of BPA over SCA in our study, in contrast to the qualitative comparability between the 2 agents as described by the authors therein. Whereas differences in thoracic versus cerebral anatomy, particularly differences in vessel size, make conclusive judgments in this respect difficult, the significance of injection rate is strongly considered, given the preferential effects of BPA on transverse (r2) over longitudinal (r1) relaxation enhancement, approaching 6:1 at 3T. ${ }^{9}$ This may be especially problematic at high field, or in large vessels such as the subclavian arteries, in which unanticipated signal loss may occur at peak bolus as the result of $\mathrm{T} 2{ }^{\star}$ effects. Dose and injection rate reductions, as well as lower field scanning, may therefore ameliorate such factors when relevant. The use of $1.5 \mathrm{~T}$ clinical systems may have further benefited this investigation, as the $\mathrm{r} 1$ and the rate of longitudinal relaxation enhancement (R1) have shown to be optimized for the macromolecular structure of BPA at $1.5 \mathrm{~T}$, whereas the differences compared with SCA are less pronounced at $3 \mathrm{~T}^{9}{ }^{9}$ In our experience, inner-thigh and midsection burning and discomfort, sometimes described on more rapid injection of BPA, are mitigated by the slower injection rate prescribed in our studies. ${ }^{21}$

Frydrychowicz et $\mathrm{al}^{13}$ estimated both SNR and contrast-tonoise ratio (CNR) between SCA and BPA; however as they point out, and as thoroughly expounded previously by Reeder et al, ${ }^{22}$ SNR and CNR measurements of multichannel receive datasets are not straightforward, given the spatial nonuniformity of noise amplification inherent to parallel acceleration. For this reason, we believe qualitative estimates of image quality and quantitative descriptions of contrast kinetics to be more relevant to clinical practice. It is worth noting, however, that their study described the inferiority of arterial SNR and CNR with BPA, again possibly relating to degradation in imaging quality related to $\mathrm{T} 2{ }^{\star}$ effects. Interestingly, their findings described a reversal of this effect dur- 
ing the venous phase, in which relative dilution of the BPA is believed to restore the benefits of greater longitudinal relaxation enhancement. Among the quantitative details considered in this study, we found no significant difference in the likelihood of the 2 agents to produce uncontaminated (ie, free of venous enhancement) arteriograms; however, as might be anticipated from the slower injection rate of BPA, the peak arterial phase was encountered significantly later than with SCA.

Although use of early formulations of blood-pool prototypes was complicated by their tendency for tissue retention, gadofosveset trisodium was the first BPA, which progressed to human trials because of its efficient excretion. ${ }^{11}$ Its high protein binding occurs almost immediately on injection, ensuring prolonged plasma half-life without extravasation-and therefore excellent blood-to-tissue contrast-while the maintenance of a predictable equilibrium between free and bound fractions ensures ready glomerular filtration of the free fraction, thus preventing retention within viscera. ${ }^{11}$ Together with the excellent relaxation enhancement properties of its macromolecular structure, the profile of gadofosveset is therefore well-suited to such applications.

Limitations of this study include its retrospective design; however, both readers were fully blinded to the administered agent before review of both the raw volumes and MIP datasets. We chose to perform consensus reads of the study data to ensure uniformity in qualitative analyses, because our primary aim was to establish the qualitative superiority of an administered agent for TR-MRA rather than specifically to investigate the comparative diagnostic accuracy of one agent against the other. Given the extremely large datasets, which were examined across broad vascular anatomic boundaries precluding reliable uniformity in data presentation, we found a consensus evaluation of angiographic quality among the proposed metrics to be most generalizable to future clinical use. This approach, unfortunately, did not permit determination of interreader variability. All data were, however, reviewed with a uniform and reproducible methodology to approximate clinical practice as closely as achievable.

Our large study population of 100 TR-MR angiographies, comprising 50 studies, each performed with either BPA or SCA, constitutes a considerably larger study sample than comparable prior investigations, ranging between 10-30 TR-MRA examinations. ${ }^{4,13,15-17,23-26}$ Not all patients were disease-free in our study population; attention was focused on the anatomically normal side for analysis, and we thus believe the findings to be readily generalizable to clinical practice and advocate strongly for the use of BPA for cerebral TR-MRA when feasible. We propose that the superior quality, comparable enhancement kinetics, and flexibility toward a 70\% dose reduction compared with SCA underscore the advantages of BPA for TR-MRA, particularly at 1.5T scanning; however, at approximately 4 times the cost per dose, supplanting standard agents for all applications may be impractical at present.

\section{CONCLUSIONS}

The present study confirms the superiority of BPA for use in cerebral TR-MRA over conventional SCA. Superior quality was noted for BPA across all interrogated arterial segments, as well as the deep venous anatomy. No significant loss in the generation of uncorrupted arteriographic phases was noted, despite the considerably smaller dose of injected gadolinium chelate with BPA. Our findings confirm not only technical feasibility but also augmented diagnostic confidence with BPA over SCA for TR-MRA, together with an advantageous dose profile in patients undergoing steadystate or dynamic MRA.

\section{REFERENCES}

1. Korosec FR, Frayne R, Grist TM, et al. Time-resolved contrast-enhanced 3D MR angiography. Magn Reson Med 1996;36:345-51

2. Lim RP, Shapiro M, Wang EY, et al. 3D time-resolved MR angiography (MRA) of the carotid arteries with time-resolved imaging with stochastic trajectories: comparison with $3 \mathrm{D}$ contrast-enhanced bolus-chase MRA and 3D time-of-flight MRA. AJNR Am J Neuroradiol 2008;29:1847-54

3. Willinek WA, Hadizadeh DR, von Falkenhausen M, et al. 4D timeresolved MR angiography with keyhole (4D-TRAK): more than 60 times accelerated MRA using a combination of CENTRA, keyhole, and SENSE at 3.0T. J Magn Reson Imaging 2008;27:1455-60

4. Maki JH, Wang M, Wilson GJ, et al. Highly accelerated first-pass contrast-enhanced magnetic resonance angiography of the peripheral vasculature: comparison of gadofosveset trisodium with gadopentetate dimeglumine contrast agents. J Magn Reson Imaging 2009;30:1085-92

5. Lin W, Haacke EM, Smith AS, et al. Gadolinium-enhanced highresolution MR angiography with adaptive vessel tracking: preliminary results in the intracranial circulation. J Magn Reson Imaging 1992;2:277-84

6. Collidge TA, Thomson PC, Mark PB, et al. Gadolinium-enhanced MR imaging and nephrogenic systemic fibrosis: retrospective study of a renal replacement therapy cohort. Radiology 2007;245:168-75

7. Caravan P, Cloutier NJ, Greenfield MT, et al. The interaction of MS325 with human serum albumin and its effect on proton relaxation rates. J Am Chem Soc 2002;124:3152-62

8. Muller RN, Gillis P, Moiny F, et al. Transverse relaxivity of particulate MRI contrast media: from theories to experiments. Magn Reson Med 1991;22:178-82

9. Rohrer M, Bauer H, Mintorovitch J, et al. Comparison of magnetic properties of MRI contrast media solutions at different magnetic field strengths. Invest Radiol 2005;40:715-24

10. Hartmann M, Wiethoff AJ, Hentrich HR, et al. Initial imaging recommendations for Vasovist angiography. Eur Radiol 2006;16(Suppl 2):B15-23

11. Lauffer RB, Parmelee DJ, Dunham SU, et al. MS-325: albumin-targeted contrast agent for MR angiography. Radiology 1998;207:529-38

12. Niendorf HP, Felix R, Laniado M, et al. Gadolinium-DTPA: a new contrast agent for magnetic resonance imaging. Radiat Med $1985 ; 3: 7-12$

13. Frydrychowicz A, Russe MF, Bock J, et al. Comparison of gadofosveset trisodium and gadobenate dimeglumine during time-resolved thoracic MR angiography at 3T. Acad Radiol 2010;17:1394-400

14. Klessen C, Hein PA, Huppertz A, et al. First-pass whole-body magnetic resonance angiography (MRA) using the blood-pool contrast medium gadofosveset trisodium: comparison to gadopentetate dimeglumine. Invest Radiol 2007;42:659-64

15. Naehle CP, Muller A, Willinek WA, et al. First-pass and steady-state magnetic resonance angiography of the thoracic vasculature using gadofosveset trisodium. J Magn Reson Imaging 2009;30:809-16

16. Nielsen YW, Eiberg JP, Logager VB, et al. Whole-body MR angiography with body coil acquisition at $3 \mathrm{~T}$ in patients with peripheral arterial disease using the contrast agent gadofosveset trisodium. Acad Radiol 2009;16:654-61

17. Nissen JC, Attenberger UI, Fink C, et al. Thoracic and abdominal 
MRA with gadofosveset: influence of injection rate on vessel signal and image quality. Eur Radiol 2009;19:1932-38

18. Bleicher AG, Kanal E. A serial dilution study of gadolinium-based MR imaging contrast agents. AJNR Am J Neuroradiol 2008;29:668-73

19. Pintaske J, Martirosian $\mathrm{P}, \mathrm{Graf} \mathrm{H}$, et al. Relaxivity of gadopentetate dimeglumine (Magnevist), gadobutrol (Gadovist), and gadobenate dimeglumine (MultiHance) in human blood plasma at 0.2, 1.5, and 3 Tesla. Invest Radiol 2006;41:213-21

20. Kiefer C, El-Koussy M, Schroth G, et al. Theoretical and clinical aspects of TWIST based 4D time-resolved MR angiography. In: Dössel O, Schlegel W, eds. World Congress on Medical Physics and Biomedical Engineering, September 7-12, 2009, Munich, Germany. Berlin/Heidelberg: Springer-Verlag; 2009:466-69

21. Rapp JH, Wolff SD, Quinn SF, et al. Aortoiliac occlusive disease in patients with known or suspected peripheral vascular disease: safety and efficacy of gadofosveset-enhanced MR angiography: multicenter comparative phase III study. Radiology 2005;236:71-78

22. Reeder SB, Wintersperger BJ, Dietrich O, et al. Practical approaches to the evaluation of signal-to-noise ratio performance with parallel imaging: application with cardiac imaging and a 32-channel cardiac coil. Magn Reson Med 2005;54:748-54

23. Bluemke DA, Stillman AE, Bis KG, et al. Carotid MR angiography: phase II study of safety and efficacy for MS-325. Radiology 2001;219:114-22

24. Hadizadeh DR, Gieseke J, Lohmaier SH, et al. Peripheral MR angiography with blood pool contrast agent: prospective intraindividual comparative study of high-spatial-resolution steady-state MR angiography versus standard-resolution first-pass MR angiography and DSA. Radiology 2008;249:701-11

25. Michaely HJ, Attenberger UI, Dietrich O, et al. Feasibility of gadofosveset-enhanced steady-state magnetic resonance angiography of the peripheral vessels at 3 Tesla with Dixon fat saturation. Invest Radiol 2008;43:635-41

26. Michaely HJ, Kramer H, Dietrich O, et al. Intraindividual comparison of high-spatial-resolution abdominal MR angiography at $1.5 \mathrm{~T}$ and 3.0 T: initial experience. Radiology 2007;244:907-13 